\title{
Pulmonary Thromboendarterectomy Without Circulatory Arrest
}

\author{
Reuben Lamiaki Kynta ${ }^{1}$, MCh; Sanjib Rawat ${ }^{1}$, MCh; Mrinal Mandal ${ }^{1}$, MSc; Manuj Kumar Saikia ${ }^{1}$, MCh
}

DOI: $10.21470 / 1678-9741-2020-0534$

\begin{abstract}
Introduction: Here we describe our technique and results of beating heart pulmonary thromboendarterectomy (PTE) with cardiopulmonary bypass (CPB) in four patients for treatment of chronic thromboembolic pulmonary hypertension (CTEPH).

Methods: Retrospective analysis of data from patients who underwent PTE for CTEPH between January 2019 and September 2020. Patients were followed up with clinical assessment, 2D echocardiography, and computed tomography pulmonary angiogram.

Results: Four patients were operated for CTEPH using our technique. Moderate tricuspid regurgitation (TR) and severe TR were found in two patients each. Severe right ventricular (RV) dysfunction was found in all cases. Thrombi were classified as Jamieson type II in three cases and type I in one case. Postoperative median direct manometric pulmonary artery (PA) pressures decreased (from $46.5 \mathrm{mmHg}$ to $23.5 \mathrm{mmHg}$ ), median CPB time was 126 minutes, and median temperature was $33.35^{\circ} \mathrm{C}$. Mechanical ventilation
\end{abstract}

was for a median of 19.5 hours. There was one re-exploration. Median intensive care unit stay was 7.5 days. There was no mortality. Postoperative $2 \mathrm{D}$ echocardiography revealed decrease in median PA systolic pressures (from $85 \mathrm{mmHg}$ to $33 \mathrm{mmHg}$ ), improvement in RV function by tricuspid annular plane systolic excursion (median $14 \mathrm{~mm}$ vs. $16 \mathrm{~mm}$ ), and improved postoperative oxygen saturations ( $88.5 \%$ vs. $99 \%$ ). In follow-up (ranging between 2-15 months), all patients reported improvement in quality of life and were in New York Heart Association class I.

Conclusion: With our described simple modifications, advances in perfusion, and blood conservation technologies, one can avoid the need for deep hypothermic circulatory arrest during PTE.

Keywords: Pulmonary Hypertension. Pulmonary Artery. Endarterectomy. Cardiopulmonary Bypass. Temperature. Oxygen. Tricuspid Valve Insufficiency. Quality of Life.

\begin{tabular}{llll}
\hline Abbreviations, acronyms \& symbols & & \\
\hline 2D echo & $=$ 2D echocardiography & mPAP & $=$ Mean pulmonary artery pressure \\
ABP & = Arterial blood pressure & NYHA & $=$ New York Heart Association Functional Classification \\
CPB & Cardiopulmonary bypass & PA & $=$ Pulmonary artery \\
CTEPH & $=$ Chronic thromboembolic pulmonary hypertension & PAP & $=$ Pulmonary artery pressure \\
CTPA & Computed tomography pulmonary angiogram & PASP & $=$ Pulmonary artery systolic pressure \\
CVP & $=$ Central venous pressure & PTE & $=$ Pulmonary thromboendarterectomy \\
DHCA & $=$ Deep hypothermic circulatory arrest & RPA & $=$ Right pulmonary artery \\
DVP & $=$ Deep venous thrombosis & RSPV & $=$ Right superior pulmonary vein \\
EF & $=$ Ejection fraction & RV & $=$ Right ventricular \\
ICU & $=$ Intensive care unit & SpO & $=$ Oxygen saturation \\
IVC & $=$ Inferior vena cava & SVC & $=$ Superior vena cava \\
LPA & $=$ Left pulmonary artery & TAPSE & $=$ Tricuspid annular plane systolic excursion \\
MAP & $=$ Mean arterial pressure & TR & $=$ Tricuspid regurgitation \\
MPA & $=$ Main pulmonary artery & UCSD & $=$ University of California San Diego \\
\hline
\end{tabular}

'Department of Cardiothoracic and Vascular Surgery, North Eastern Indira Gandhi Regional Institute of Health and Medical Sciences, Shillong, Meghalaya, India.

This study was carried out at the Department of Cardiothoracic and Vascular Surgery, North Eastern Indira Gandhi Regional Institute of Health and Medical Sciences, Shillong, Meghalaya, India.
Correspondence Address:

Reuben Lamiaki Kynta

(iD) https://orcid.org/0000-0002-7640-180X

Department of Cardiothoracic and Vascular Surgery, North Eastern Indira Gandhi

Regional Institute of Health and Medical Sciences

Shillong, Meghalaya, India - Zip Code: 793018

E-mail: reuben.kynta@gmail.com 


\section{INTRODUCTION}

Pulmonary thromboendarterectomy (PTE) is the definitive surgical treatment for chronic thromboembolic pulmonary hypertension $(\mathrm{CTEPH})^{[1]}$. In this technique, a bloodless field is essential, and deep hypothermic circulatory arrest (DHCA) has been the preferred method to extract the pulmonary thrombus beyond the sublobar levels. However, DHCA is not devoid of its adverse effects which mainly include neurologic deficits ${ }^{[2,3]}$, renal dysfunction ${ }^{[4,5]}$, and bleeding ${ }^{[6]}$. In pursuit to control the troublesome back bleeding from the bronchial vessels and collaterals without the use of DHCA, several methods have been described to achieve complete thromboendarterectomy. Here, we describe our technique and modifications needed for PTE on beating heart cardiopulmonary bypass (CPB) with mild hypothermia on four patients.

\section{METHODS}

\section{Study Design}

As this was a retrospective case series, ethical clearance was waived off. Retrospective data of patients who underwent beating heart PTE from January 2019 to September 2020 at the Department of Cardiothoracic and Vascular Surgery, North Eastern Indira Gandhi Regional Institute of Health and Medical Sciences (Shillong, India), were collected, and the operative records were reviewed. This study included four patients. Written and informed consent was taken. The patients were followed up with clinical assessment, investigations like transthoracic 2D echocardiography (2D echo) and computed tomography pulmonary angiogram (CTPA) were performed, and the data was collected.

Inclusion criteria were patients reporting to/referred to our department with: (a) New York Heart Association Functional Classification (NYHA) class III \& IV symptoms, (b) thrombus in main, lobar, or segmental pulmonary arteries (PA) as seen in CTPA, (c) no debilitating comorbidities, and (d) 2D echo evidence of right ventricular (RV) dysfunction. All patients with acute pulmonary embolism did not fit to undergo CPB, and those for emergency pulmonary embolectomy were excluded.

Demographic variables, pulmonary artery pressure (PAP) (systolic, diastolic, and mean), RV dysfunction as measured by tricuspid annular plane systolic excursion (TAPSE) on 2D echo, central venous pressure (CVP), oxygen saturation $\left(\mathrm{SpO}_{2}\right)$, CPB time, location of thrombus as described by Jamieson's classification as well as University of California San Diego (UCSD) surgical classification, postoperative course, and any postoperative complications were studied.

Pulmonary artery systolic pressure (PASP) was measured by pre- and postoperative echocardiogram. PAP was measured intraoperatively before and after PTE by direct needle manometry of main pulmonary artery (MPA). CVP and $\mathrm{SpO}_{2}$ were measured before and after PTE.

\section{Anesthetic Management}

Anesthesia was induced by intravenous administration of morphine, fentanyl, and vecuronium and maintained with intravenously administered propofol and inhalation of isoflurane. Monitoring was done by a radial artery line, central venous line, electrocardiography, nasopharyngeal and rectal thermometer, and transesophageal echocardiography. The heart rate was regulated to 60-70 beats per minute by boluses of beta blocker metoprolol.

\section{CPB Pump and Autologous Blood Conservation Setup}

Standard CPB circuit with St. Thomas II cardioplegia on standby and a cell saver (C.A.T.S ${ }^{\circledR}$, Fresenius Kabi) is established. An aortic cannula and two straight venous cannulae for bicaval cannulation of appropriate size are used. Four pump suction lines are readied for a free cardiotomy sucker, vent sucker of the right superior pulmonary vein (RSPV), right ventricle, and either of the PAs (Figures 1 and 2). A hemoconcentrator for ultrafiltration is connected to the bypass circuit to achieve negative fluid balance. Core temperature is decreased to $32-34{ }^{\circ} \mathrm{C}$ (mild hypothermia), and flows are maintained at 2 to $2.4 \mathrm{l} / \mathrm{min} / \mathrm{m}^{2}$. A high-pressure external sucker attached to the cell saver is used to connect an olive tip sucker, which is used as a dissector. The a-stat strategy of $\mathrm{pH}$ management is employed.

\section{Instruments Required}

Two DeBakey vascular forceps (23 cm length), two ventricular septal defect retractors (23 cm length), one olive tip sucker connected to cell saver, a mastoid retractor with rubber inserts

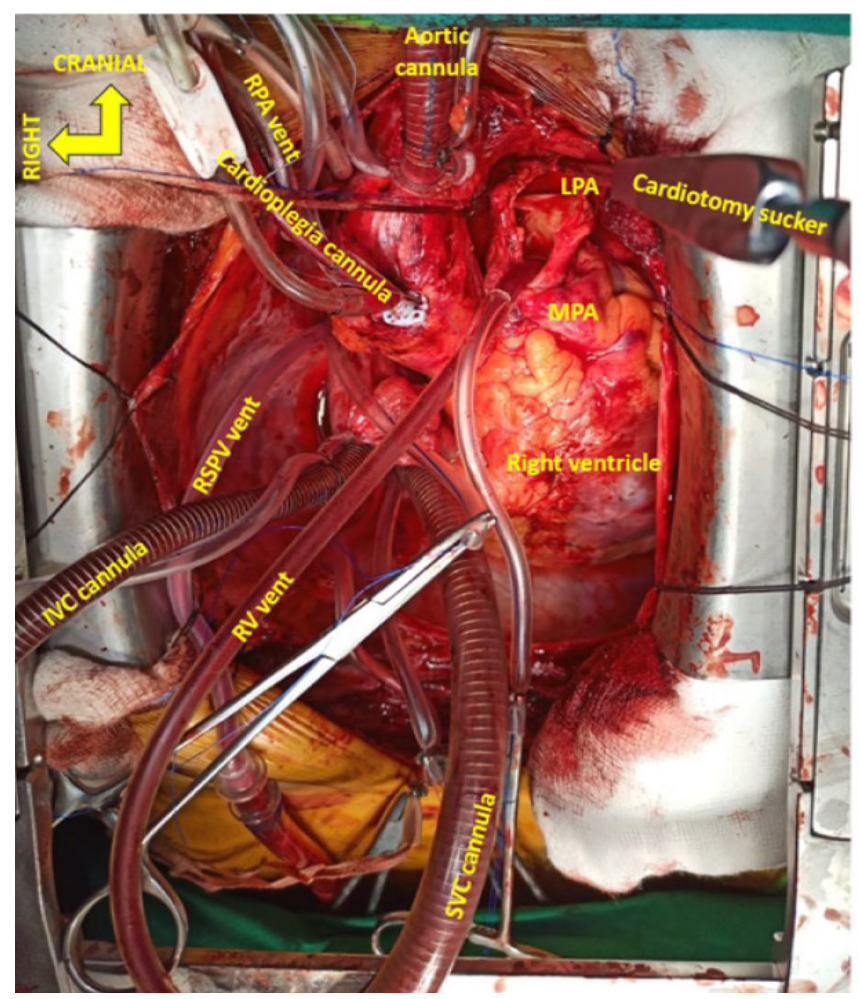

Fig. 1 - Cannulae and vent setup. Main pulmonary artery (MPA) arteriotomy extending to left pulmonary artery (LPA) with cardiotomy sucker in LPA shown. IVC=inferior vena cava; RPA=right pulmonary artery; $R V=$ right ventricular; $R S P V=$ right superior pulmonary vein; SVC=superior vena cava 


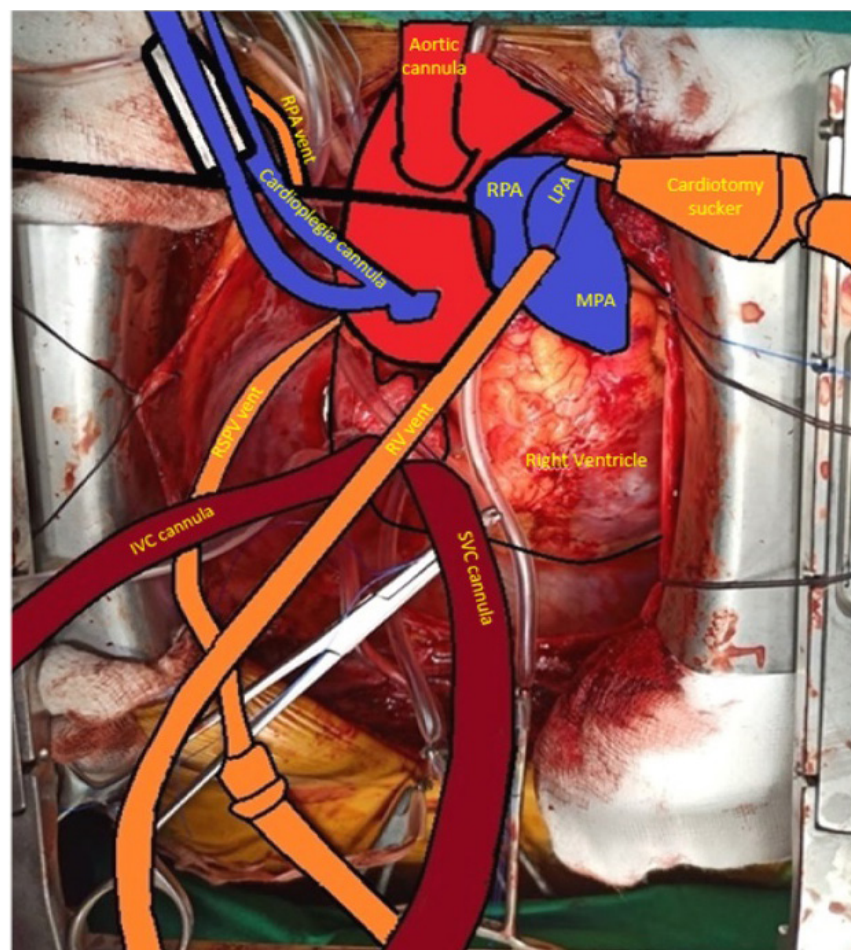

Fig. 2 - Cartoon of cannulae and vent setup. IVC=inferior vena cava; $L P A=$ left pulmonary artery; $M P A=$ main pulmonary artery; $R P A=$ right pulmonary artery; $R V=$ right ventricular; $R S P V=r i g h t$ superior pulmonary vein; $S V C=$ superior vena cava

on blade tips, and a vascular clamp (straight or angled) are required (Figure 3 ).

\section{Surgical Technique}

The operation is performed via a median sternotomy. After an inverted $T$ pericardiotomy and attachment to the wound

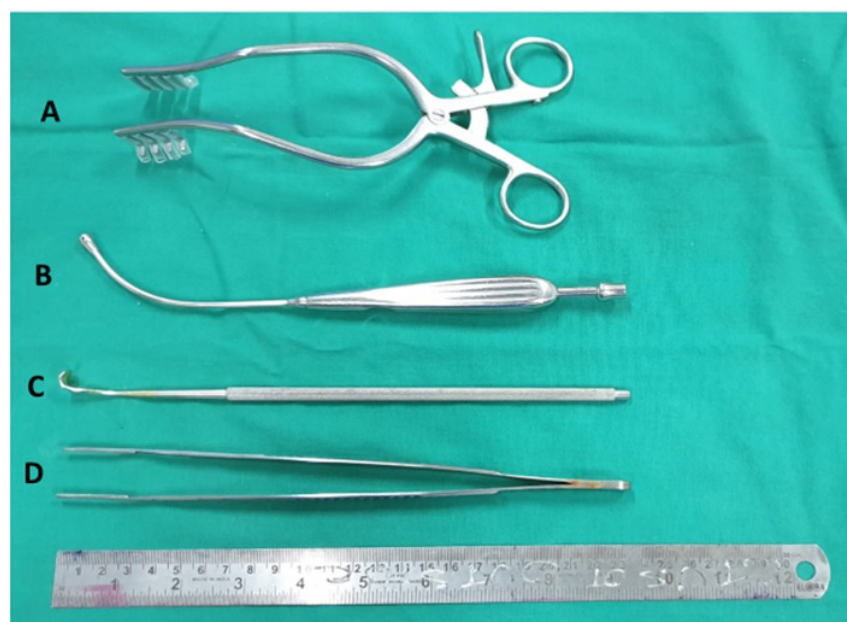

Fig. 3 - Instruments required: A) Mastoid retractor with rubber inserts on blade tips; B) Olive tip sucker; C) Ventricular septal defect retractors (23 cm length); D) DeBakey vascular forceps (23 cm length). edges, direct pressure manometry by puncturing MPA is done. The superior vena cava (SVC) is looped and mobilized by cautery dissection. Mobilization of the branch PA is done intrapericardially, and none of the pleurae are opened. The aorta is looped with a vessel loop for better visualization (Figures 1 and 2).

CPB is established by cannulating the ascending aorta and both caval veins after achieving an activated clotting time of $>$ 480 seconds with heparinization (300 U/kg).

A straight cannula in the SVC is guided high, near to the convergence of the innominate vein via the right atrial appendage, and snared. This method of cannulation further helps in proximal mobilization of the SVC. The inferior vena cava is cannulated and snared. An antegrade aortic root cardioplegia needle is inserted should the need for DHCA arise.

A vent catheter is introduced into the RSPV. The left pulmonary artery (LPA) is then dissected beyond the origin of the upper lobe artery with careful preservation of the left phrenic nerve. A small arteriotomy is made in the right pulmonary artery (RPA) just right to the aorta, and a vent catheter is introduced into it and snared. Ventilation is then stopped.

With the surgeon standing on the right side of the patient, an incision is then made in the MPA and is extended into the LPA beyond the origin of the upper lobe artery. Stay sutures are taken and the arteriotomy is exposed. A sump sucker is introduced into the right ventricle via the pulmonary valve and snared through the MPA wall. If bleeding from the RPA obscure the field of view, a vascular clamp may be applied upstream to the RPA vent.

Any fresh or loose clots are removed for visualization of the chronic thrombus. Thromboendoarterectomy then commences with creation of the correct plane in the media. The correct plane appears as a smooth pearly white structure which is amenable to peeling with controlled traction, countertraction, and a sweeping motion of the olive tipped sucker (Figure 4). The free cardiotomy sucker is employed to clear blood from the

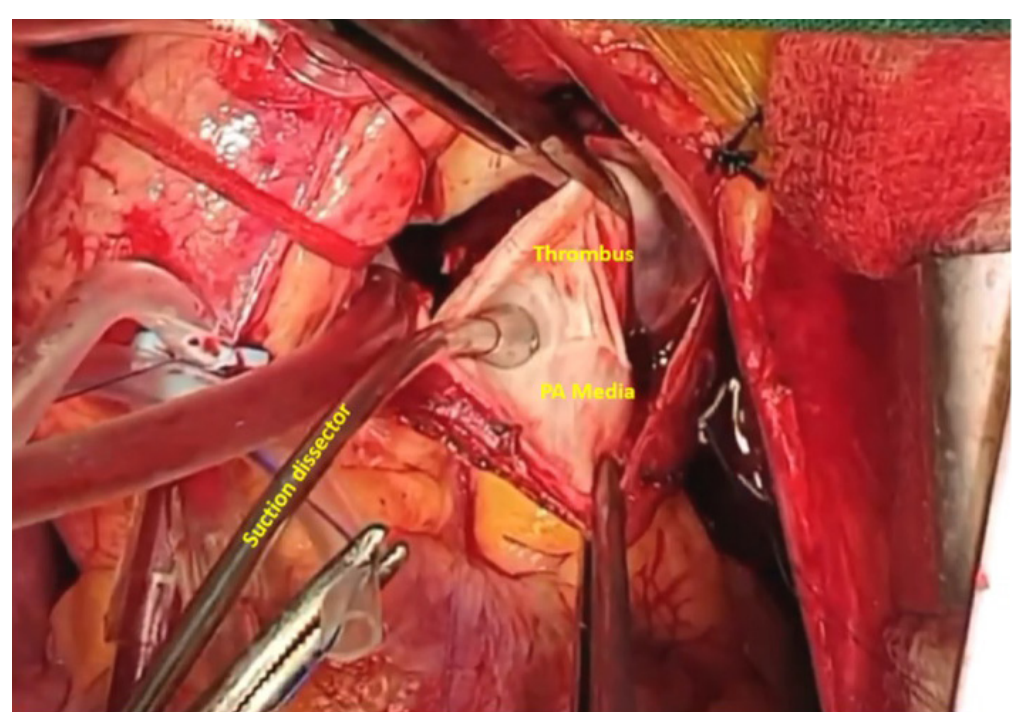

Fig. 4 - Delineation of the true plane of dissection in pulmonary thromboendarterectomy. $P A=$ pulmonary artery. 
Table 1. Perioperative details of patients undergoing beating heart pulmonary thromboendarterectomy.

\begin{tabular}{|c|c|c|c|c|c|}
\hline \multicolumn{6}{|c|}{ PREOPERATIVE FINDINGS } \\
\hline & Patient 1 & Patient 2 & Patient 3 & Patient 4 & Median \\
\hline Age (years) & 23 & 24 & 46 & 47 & 35 \\
\hline Sex & Male & Male & Female & Male & \\
\hline Preoperative ICU admission & - & - & - & - & \\
\hline DVT & - & - & - & - & \\
\hline NYHA class & III & IV & III & IV & \\
\hline \multicolumn{6}{|l|}{ 2D echo } \\
\hline EF (\%) & 60 & 60 & 57 & 60 & $60 \%$ \\
\hline PASP (mmHg) & 72 & 68 & 98 & 120 & $85 \mathrm{mmHg}$ \\
\hline TR & Severe & Moderate & Severe & Moderate & \\
\hline TAPSE (mm) & 15 & 18 & 13 & 13 & $14 \mathrm{~mm}$ \\
\hline \multicolumn{6}{|l|}{ Preoperative CTPA } \\
\hline RPA, mm & 16 & *29 & *21 & 35 & \\
\hline LPA, mm & *19 & *26 & *23 & *33 & \\
\hline MPA, mm & *24 & 40 & 34 & *39 & \\
\hline \multicolumn{6}{|c|}{ INTRAOPERATIVE FINDINGS } \\
\hline Jamieson class & $\|$ & । & ॥ & II (LPA only) & \\
\hline UCSD surgical class & I & I & I & IC left & \\
\hline CPB time (minutes) & 108 & 117 & 135 & 161 & 126 \\
\hline Minimum temp $\left({ }^{\circ} \mathrm{C}\right)$ & 28 & 33.9 & 33.7 & 33 & 33.35 \\
\hline \multicolumn{6}{|l|}{$\mathrm{SpO}_{2}(\%)$} \\
\hline Preoperative & 89 & 79 & 88 & 93 & 88.5 \\
\hline Postoperative & 99 & 99 & 100 & 99 & 99 \\
\hline \multicolumn{6}{|l|}{ CVP } \\
\hline Preoperative & 25 & 22 & 19 & 26 & 23.5 \\
\hline Postoperative & 13 & 12 & 12 & 11 & 12 \\
\hline \multicolumn{6}{|c|}{ ABP (systolic/diastolic/mean in $\mathrm{mmHg}$ ) } \\
\hline Preoperative & $98 / 60 / 73$ & $78 / 40 / 52$ & $74 / 49 / 57$ & $145 / 110 / 122$ & MAP 65 \\
\hline Postoperative & 118/82/94 & $78 / 51 / 60$ & $116 / 69 / 85$ & $131 / 51 / 78$ & MAP 81.5 \\
\hline \multicolumn{6}{|c|}{ PAP (systolic/diastolic/mean in $\mathrm{mmHg}$ ) } \\
\hline Preoperative & $65 / 34 / 44$ & $51 / 48 / 49$ & 24/15/18 & $104 / 49 / 67$ & mPAP 46.5 \\
\hline Postoperative & $35 / 20 / 25$ & $27 / 20 / 22$ & 28/17/21 & $40 / 26 / 31$ & mPAP 23.5 \\
\hline \multicolumn{6}{|c|}{ POSTOPERATIVE FINDINGS } \\
\hline Re-exploration & No & No & No & Yes & \\
\hline Ventilation (hours) & 24 & 15 & 12 & 96 & 19.5 \\
\hline ICU stay (days) & 4 & 3 & 11 & 14 & 7.5 \\
\hline Hospital stay (days) & 23 & 29 & 22 & 40 & 26 \\
\hline NYHA class & 1 & 1 & I & 1 & \\
\hline \multicolumn{6}{|l|}{ 2D echo } \\
\hline EF (\%) & 58 & 60 & 60 & 65 & $60 \%$ \\
\hline
\end{tabular}




\begin{tabular}{l|c|c|c|c|c}
\hline PASP $(\mathrm{mmHg})$ & 30 & 25 & 40 & 36 & $33 \mathrm{mmHg}$ \\
\hline TR & Trace & Trace & Moderate & Mild & \\
\hline TAPSE $(\mathrm{mm})$ & 20 & 16 & 15 & 16 & $16 \mathrm{~mm}$ \\
\hline
\end{tabular}

\section{Postoperative CTPA}

\begin{tabular}{l|l|l|l|l|l}
\hline RPA, $\mathrm{mm}$ & 18 & 19 & 17 & 24 & 18 \\
\hline LPA, $\mathrm{mm}$ & 22 & 10 & 20 & 33 & 34 \\
\hline MPA, mm & 28 & 35 & 4 & 2 & 9 \\
\hline Follow-up (months) & 15 & 14 & & \\
\hline
\end{tabular}

*Thrombus

2D echo=2D echocardiography; $\mathrm{ABP}=$ arterial blood pressure; $\mathrm{CPB}=$ cardiopulmonary bypass; $C \mathrm{TPA}=$ computed tomography pulmonary angiogram; CVP=central venous pressure; DVT=deep venous thrombosis; $E F=e j e c t i o n$ fraction; ICU=intensive care unit; LPA=left pulmonary artery; $M A P=$ mean arterial pressure (in $\mathrm{mmHg}$ ); $\mathrm{MPA}=$ main pulmonary artery; $\mathrm{mPAP}=\mathrm{mean}$ pulmonary artery pressure (in $\mathrm{mmHg}$ ); NYHA=New York Heart Association Functional Classification; PAP=pulmonary artery pressure (by needle manometry); $\mathrm{PASP}=$ pulmonary artery systolic pressure; $\mathrm{RPA}=$ right pulmonary artery; $\mathrm{SpO}_{2}=$ oxygen saturation; TAPSE=tricuspid annular plane systolic excursion; TR=tricuspid regurgitation; UCSD=University of California San Diego

PTE[11-13]. Our method of PTE differs from the method described by Mikus et al. ${ }^{[14]}$ in the fact that we use extensive venting of the left atrium via the RSPV, the right ventricle, the contralateral PA, and do not arrest the heart. We, however, follow a strategy of blood conservation similar to the described by the author by using a negative suction dissector connected to a cell saver. We also differ from the technique described by Bisoi et al. ${ }^{[15]}$ because we vent the RSPV and do not transect the SVC.

In our series, the hospital stay was prolonged because we are located in a remote area with poor patient compliance on follow-up. Our low-cost modifications for the conduct of PTE in this resource poor region are unique because of the use of mild hypothermia, the avoidance of cardioplegic arrest, avoidance of transection of the great vessels, and the ability to obtain a relatively bloodless field using external negative suction. The thorough checking of clearance the endarterectomized field by an endoscope also ensures that we can address remaining thrombus burden in the same setting.

\section{Limitations}

This study has its limitations. First, it was a retrospective analysis covering a 20-month period. Second, small sample size is a limiting factor to gain significant results. However, we believe this study may provide some useful information for the surgical management of CTEPH patients

\section{CONCLUSION}

The definitive treatment of chronic pulmonary hypertension is the complete evacuation of the thrombus load in the pulmonary vascular tree. Thromboendarterectomy under DHCA has been the most commonly technique employed to omit bronchial back bleed. Advances in perfusion, blood conservation, and our described modifications can make it possible to avoid circulatory arrest. However, the superiority of our technique cannot be established in view of the small number of cases.

\section{No financial support.}

No conflict of interest.
MM
Authors' Roles \& Responsibilities

RLK Substantial contributions to the conception or design of the work; or the acquisition, analysis, or interpretation of data for the work; drafting the work or revising it critically for important intellectual content; final approval of the version to be published

SR

Drafting the work or revising it critically for important intellectual content; agreement to be accountable for all aspects of the work in ensuring that questions related to the accuracy or integrity of any part of the work are appropriately investigated and resolved; final approval of the version to be published

Agreement to be accountable for all aspects of the work in ensuring that questions related to the accuracy or integrity of any part of the work are appropriately investigated and resolved; final approval of the version to be published

KS Final approval of the version to be published

\section{REFERENCES}

1. Jamieson SW, Kapelanski DP, Sakakibara N, Manecke GR, Thistlethwaite PA, Kerr KM, et al. Pulmonary endarterectomy: experience and lessons learned in 1,500 cases. Ann Thorac Surg. 2003;76(5):1457-62; discussion 1462-4. doi:10.1016/s00034975(03)00828-2.

2. Kamenskaya OV, Cherniavsky AM, Klinkova AS, Cherniavsky MA, Meshkov IO, Lomivorotov W, et al. Efficiency of various cerebral protection techniques used during the surgical treatment of chronic pulmonary thromboembolism. J Extra Corpor Technol. 2015;47(2):95-102.

3. Tian Tian DH, Wan B, Bannon PG, Misfeld M, LeMaire SA, Kazui T, et 
al. A meta-analysis of deep hypothermic circulatory arrest versus moderate hypothermic circulatory arrest with selective antegrade cerebral perfusion. Ann Cardiothorac Surg. 2013;2(2):148-58. doi:10.3978/j.issn.2225-319X.2013.03.13.

4. Yu L, Gu T, Zhang G, Cheng S, Fang Q, Mao N. The deep hypothermic circulatory arrest causes more kidney malfunctions based on a novel rabbit model. Ann Saudi Med. 2014;34(6):532-40. doi:10.5144/0256-4947.2014.532.

5. Zhang C, Wang G, Zhou H, Lei G, Yang L, Fang Z, et al. Preoperative platelet count, preoperative hemoglobin concentration and deep hypothermic circulatory arrest duration are risk factors for acute kidney injury after pulmonary endarterectomy: a retrospective cohort study. J Cardiothorac Surg. 2019;14(1):220.

6. Mazzeffi M, Marotta M, Lin HM, Fischer G. Duration of deep hypothermia during aortic surgery and the risk of perioperative blood transfusion. Ann Card Anaesth. 2012;15(4):266-73.

7. Jenkins D, Madani M, Fadel E, D'Armini AM, Mayer E. Pulmonary endarterectomy in the management of chronic thromboembolic pulmonary hypertension. Eur Respir Rev. 2017;26(143):160111. doi:10.1183/16000617.0111-2016.

8. Sakurai Y, Takami Y, Amano K, Higuchi Y, Akita K, Noda M, et al. Predictors of outcomes after surgery for chronic thromboembolic pulmonary hypertension. Ann Thorac Surg. 2019;108(4):1154-61. doi:10.1016/j.athoracsur.2019.03.100.

9. Jamieson SW. Historical perspective: surgery for chronic thromboembolic disease. Semin Thorac Cardiovasc Surg. 2006;18(3):218-22. doi:10.1053/j.semtcvs.2006.09.007.
10. Hagl C, Khaladj N, Peters T, Hoeper MM, Logemann F, Haverich A, et al. Technical advances of pulmonary thromboendarterectomy for chronic thromboembolic pulmonary hypertension. Eur J Cardiothorac Surg. 2003;23(5):776-81; discussion 781. doi:10.1016/ s1010-7940(03)00029-0.

11. Masuda M, Mogi K, Nakaya M, Pearce $Y$, Imamaki M, Shimura H, et al. Surgical treatment for chronic pulmonary thromboembolism under cardiopulmonary bypass with selective cerebral perfusion. Surg Today. 2001;31(2):108-12.

12. Thomson B, Tsui SS, Dunning J, Goodwin A, Vuylsteke A, Latimer R, et al. Pulmonary endarterectomy is possible and effective without the use of complete circulatory arrest--the UK experience in over 150 patients. Eur J Cardiothorac Surg. 2008;33(2):157-63. doi:10.1016/j. ejcts.2007.11.009.

13. Lafci G, Tasoglu I, Ulas MM, Yalcinkaya A, Cagli K. Pulmonary endarterectomy: with use of moderate hypothermia and antegrade cerebral perfusion without circulatory arrest. Tex Heart Inst J. 2012;39(1):65-7.

14. Mikus PM, Dell'Amore A, Pastore S, Galiè N. Pulmonary endarterectomy: is there an alternative to profound hypothermia with cardiocirculatory arrest? Eur J Cardiothorac Surg. 2006;30(3):563-5. doi:10.1016/j.ejcts.2006.06.016.

15. Bisoi AK, Ramakrishnan P, Chauhan S, Sahu MK, Chandrasekharan NC. An experience on normothermic beating heart pulmonary thromboendarterectomy. W J Cardiovasc Surg. 2019;9(1):1-3. doi:10.4236/wjcs.2019.91001. 